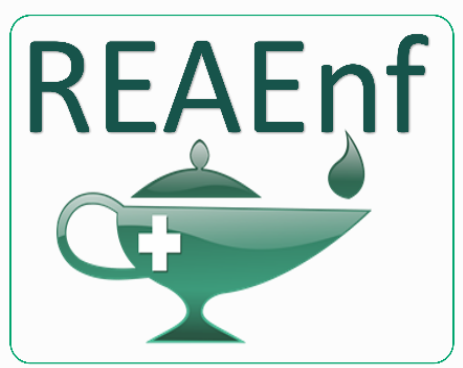

Revista Eletrônica Acervo Enfermagem
RELATO DE EXPERIÊNCIA

Recebido em: 11/2020

Aceito em: $12 / 2020$

Publicado em: 1/2021

\title{
Procedimentos e técnicas de enfermagem realizadas durante a pandemia de COVID-19
}

\author{
Nursing procedures and techniques performed during the COVID-19 pandemic
}

\section{Procedimientos y técnicas de enfermería realizadas durante la pandemia del COVID-19}

\begin{abstract}
Italo Everton Bezerra Barbosa ${ }^{1 *}$, Alicia Ribeiro Fonseca ${ }^{1}$, Francisca Crispim de Souza ${ }^{1}$, Emmanuele Neuza Moreira de Andrade1, Meire Cristina Soares Ribeiro'.
\end{abstract}

\begin{abstract}
Resumo: Esse artigo buscou descrever as experiências vivenciadas durante a realização de aulas práticas de enfermagem, durante a pandemia de COVID-19, ministradas no segundo semestre de 2020 em uma universidade particular de Manaus, capital do estado do Amazonas. Trata-se de um estudo descritivo, utilizando o método hipotético-dedutivo, realizado a partir da vivência de acadêmicos de enfermagem, durante a reposição de aulas práticas na disciplina de Semiologia e Semiotécnica. Frente a pandemia de COVID-19, algumas universidades particulares optaram pela realização das aulas teóricas do primeiro semestre à distância, priorizando se prepararem para a reposição das aulas práticas no segundo semestre, visando a prevenção de alunos e funcionários da instituição. Diante disso, as práticas realizadas durante a pandemia de COVID-19, foram de extrema importância para o desenvolvimento profissional dos acadêmicos como futuro profissionais de enfermagem. Uma vez que, essa experiência possibilitou uma oportunidade de absorver uma gama de novos conhecimentos e medidas preventivas diante essa situação.
\end{abstract}

Palavras-chave: Covid-19, Pandemia, Enfermagem.

\begin{abstract}
This article sought to describe the experiences lived during the realization of practical nursing classes, during the COVID-19 pandemic, given in the second semester of 2020 at a private university of Manaus, capital of the state of Amazonas. This is a descriptive study, using the hypothetical-deductive method, carried out from the experience of nursing students, during the replacement of practical classes in the discipline of Semiology and Semiotechnics. Faced with the COVID-19 pandemic, some private universities opted to take the theoretical classes of the first semester of at a distance, giving priority to preparing for the replacement of practical classes in the second semester, aiming at the prevention of students and employees of the institution. In view of this, the practices carried out during the COVID-19 pandemic were extremely important for the professional development of academics as future nursing professionals. Since this experience provided an opportunity to absorb a range of new knowledge and preventive measures in the face of this situation.
\end{abstract}

Keyword: Covid-19, Pandemic, Nursing.

\footnotetext{
${ }^{1}$ Centro Universitário Fametro (FAMETRO) Manaus - AM.

*E-mail: italoeverton1998@gmail.com
} 
Resumen: Este artículo buscó describir las experiencias vividas durante la realización de las clases prácticas de enfermería, durante la pandemia del COVID-19, impartidas en el segundo semestre de 2020 en una universidad privada de Manaus, capital del estado de Amazonas. Se trata de un estudio descriptivo, utilizando el método hipotético-deductivo, realizado a partir de la experiencia de estudiantes de enfermería, durante la sustitución de clases prácticas en la disciplina de Semiología y Semiotecnia. Ante la pandemia del COVID-19, algunas universidades privadas optaron por cursar las clases teóricas del primer semestre de a distancia, priorizando la preparación para la sustitución de las clases prácticas en el segundo semestre, con el objetivo de la prevención de estudiantes y empleados de la institución. Ante esto, las prácticas realizadas durante la pandemia del COVID-19 fueron de suma importancia para el desarrollo profesional de los académicos como futuros profesionales de enfermería. Desde entonces, esta experiencia brindó la oportunidad de absorber una serie de nuevos conocimientos y medidas preventivas ante esta situación.

Palabra clave: Covid-19, Pandemia, Enfermería.

\section{INTRODUÇÃO}

Em 30 de janeiro de 2020, a Organização Mundial da Saúde (OMS) declarou que, o surgimento da doença causada pelo novo coronavírus se tornou uma emergência de Saúde Pública em âmbito mundial. Em 11 de março de 2020, a OMS caracterizou a COVID-19 como uma pandemia por conta da sua rápida disseminação no mundo (CARVER PE e PHILLIPS J, 2020).

A pandemia de COVID-19 constitui um tópico novo e bastante desafiador à sociedade atual, está ocasionando modificações econômicas, políticas e principalmente social, tendo em vista, as mudanças e medidas preventivas que foram tomadas referentes ao novo coronavírus. Com milhões de casos identificados ao redor do mundo, disseminação rápida do vírus e ausência de vacinas e medicamentos eficazes para o seu tratamento, a medida mais segura tanto para prevenção e propagação do vírus ainda é isolamento social (HEYMANN DL, et al., 2020).

Foram estabelecidas algumas ações e estratégias pela OMS, na tentativa de interromper a transmissão do vírus de humano para humano, uma vez que, o vírus possui uma disseminação bastante rápida e ainda não existe uma vacina contra o novo coronavírus. Diante disso, as principais medidas estabelecidas foram identificar as pessoas contaminadas, isolar e cuidar dos pacientes infectados, tentar diminuir a disseminação do vírus evitando a aglomeração de pessoas, realizando campanhas de conscientização sobre a importância do uso da máscara e utilização do álcool em gel (OMS BR, 2020).

Diante dessa situação, em março de 2020, as escolas e universidades brasileiras tiveram que suspender todas as atividades que envolviam aglomerações de pessoas, independentemente do local, devido à pandemia de COVID-19. Como isso, as instituições tiveram que tentar encontrar uma solução para que os alunos não se prejudicassem em relação a paralização das aulas. Na tentativa de solucionar os problemas e se adequar à situação atual, as universidades optaram por aulas remotas (SEWART D, et al., 2020).

Relativo a essa situação, os professores visando atender a situação atual em que o mundo se encontra, optaram por estratégias utilizando as metodologias a distância, tendo em vista que, o assunto referente ao novo coronavírus era algo novo e as instituições de ensino ainda não estavam preparadas para enfrentar essa situação. Outro ponto importante, foi a deficiência que muitos professores apresentaram em relação ao contato com o uso de novas temáticas, utilizando a tecnologia como novo método para passar o conhecimento aos alunos (GOULART MB, et al., 2018).

Com a chegada da pandemia de COVID-19, e o isolamento social já sendo colocado em vigor, o fechamento das escolas e universidades era algo que já estava previsto acontecer, considerando a falta de itens e equipamentos de proteção individual as universidades optaram por dar início as aulas remotas e se preparar para a volta das aulas presenciais no segundo semestre de 2020 (SILVA AAM, 2020).

No meio dessa nova situação, em que tanto os professores quanto os alunos não estavam preparados para atuarem neste cenário de pandemia, muitas fragilidades foram encontradas referente ao novo método 
de ensino. Dessa maneira, o acesso remoto apresenta algumas complicações e instabilidades de acesso e de manuseio para tanto para os professores e alunos (ABEN BR, 2020).

Nesse momento, no que se diz respeito a educação, as atividades a distancias estavam sendo planejadas de modo emergencial, para tentar não deixar os alunos prejudicados em relação ao tempo e conteúdo. Deste modo, torna-se importante destacar que ensino remoto em conjunto com a educação à distância são modalidades que possuem apresentações diferentes, porém possui as mesmas metodologias e objetivos (ALVES A, 2011).

Dificilmente algum recurso irá substituir o ensino presencial, mesmo existindo várias possibilidades de troca de conhecimento. É certo que o ensino a distância e um método novo, que muitas pessoas não estavam preparadas para Ihe dar com isso. Porém, na presente situação em que o mundo se encontra, as aulas remotas ou pelo menos uma parte delas, ainda é a melhor solução para tentar diminuir a disseminação do vírus (GOULART BHE, et al., 2018).

Diante disso, o presente artigo objetiva descrever as experiências vivenciadas durante a reposição de aulas práticas de enfermagem, ministradas no segundo semestre de 2020 em uma Universidade particular do Amazonas, durante a pandemia de COVID-19. Tendo em vista que, a instituição tomou todas as medidas de prevenção possíveis para evitar a disseminação do vírus, seguindo as recomendações da Organização Mundial de Saúde (OMS).

\section{RELATO DE EXPERIÊNCIA}

Trata-se de um estudo descritivo, do tipo relato de experiência, realizado a partir da vivência de acadêmicos de em enfermagem, referente a reposição de aulas práticas na disciplina de Semiologia e Semiotécnica, oferecidas por uma universidade particular, localizado na capital do estado do Amazonas

O presente trabalho utilizou o método hipotético-dedutivo, em conjunto com o tipo de pesquisa descritiva, que visa descrever as características de uma determinada área, fenômeno ou população. Utilizando técnicas padronizadas, direcionadas a coleta de dados, em que se tenha relações entre variáveis do mesmo seguimento, utilizando questionários e observação sistemática ao fenômeno em que se quer realizar a pesquisa (GIL A, 2010).

Relativo à pandemia de COVID-19, as universidades particulares, priorizaram realizar as aulas no primeiro semestre de 2020 de forma remota, ou seja, a distância, uma vez que a propagação do novo coronavírus ainda era bastante grande. Optaram por continuar o cronograma, porém, realizando algumas mudanças no plano de ensino-aprendizagem dos alunos.

Nessa situação, as aulas práticas que estavam previstas acontecer no primeiro semestre do ano letivo, foram adiadas e realizadas no segundo semestre, as atividades práticas ocorreram de forma bastante diferente do que era realizado no ano anterior. Para que as práticas fossem realizadas seria necessária uma atenção maior por parte da instituição e dos alunos, para que as atividades pudessem ser realizadas da forma mais segura possível, seguindo as medidas de prevenção pela OMS.

Para que os alunos pudessem participar das aulas práticas, os mesmos deveriam dispor de alguns materiais e equipamentos de proteção individual para sua prevenção e de todos os outros alunos. A turma continha um total 48 alunos, desse modo a melhor solução foi dividir a turma por grupos para que as práticas fossem compreendidas da melhor forma possível, evitando assim também possíveis aglomerações por parte dos discentes.

Foi elaborado um quadro, contendo a divisão dos alunos, os dias que cada grupo iria realizar suas práticas e qual atividade seria realizada naquele dia, cada aluno poderia ir somente no horário estipulado pelo professor, conforme a instituição disponibilizava o uso do laboratório. A turma foi dividida em 4 grupos de 12 alunos, as atividades práticas ocorram no decorre do mês de agosto, conforme apresentado abaixo no Quadro 1. 
Quadro 1 - Divisão das atividades por grupos.

\begin{tabular}{|c|c|c|c|c|}
\hline \multirow{2}{*}{ Práticas de enfermagem } & $\begin{array}{l}\text { 1-Semana } \\
\text { 1-Grupo }\end{array}$ & $\begin{array}{l}\text { 2-Semana } \\
\text { 2-Grupo }\end{array}$ & $\begin{array}{l}\text { 3-Semana } \\
\text { 3-Grupo }\end{array}$ & $\begin{array}{c}\text { 4-Semana } \\
\text { 4-Grupo }\end{array}$ \\
\hline & \multicolumn{4}{|c|}{ Dias de práticas } \\
\hline $\begin{array}{l}\text { Higienização } \\
\text { das mãos }\end{array}$ & 3 & 10 & 17 & 24 \\
\hline $\begin{array}{c}\text { Cálculo, diluição e } \\
\text { administração de } \\
\text { medicamentos }\end{array}$ & 4 & 12 & 18 & 25 \\
\hline $\begin{array}{c}\text { Aplicação de medicamentos } \\
\text { IM, EV, Vasto L, VG, } \\
\text { Subcutânea }\end{array}$ & 5 & 12 & 19 & 26 \\
\hline $\begin{array}{c}\text { Sondagem vesical de demora e } \\
\text { de alívio }\end{array}$ & 6 & 13 & 20 & 27 \\
\hline $\begin{array}{c}\text { Cateter nasogástrico e } \\
\text { nasoenteral }\end{array}$ & 7 & 14 & 21 & 28 \\
\hline
\end{tabular}

Fonte: Barbosa IEB, et al., 2020.

O quadro foi disponibilizado para os alunos com algumas semanas de antecedência para que os mesmos pudessem se programar referente a comprar dos materiais e equipamentos de proteção individual. Durante as aulas práticas era obrigatório a utilização de todos os equipamentos de proteção, tendo em vista que a universidade sempre era muito criteriosa e atenta em relação a proteção de todos os alunos referente a COVID-19. Os equipamentos solicitados estão apresentados no Quadro 2.

Quadro 2 - Equipamentos solicitados para realizam das práticas.

\begin{tabular}{|c|c|c|}
\hline \multicolumn{2}{|c|}{ Equipamento de proteção individual (EPI) } \\
\hline Itens & Quantidade & \multicolumn{1}{|c|}{ Finalidade } \\
\hline Luvas de procedimento & 8 & \multirow{2}{*}{$\begin{array}{l}\text { Prevenir os alunos de terem contato } \\
\text { Máscara }\end{array}$} \\
\hline Toucas & 4 & com o vírus, evitando assim também \\
\hline Jaleco & 4 & \\
\hline Viseira & 1 & \\
\hline Capote & 1 & \\
\hline
\end{tabular}

Fonte: Barbosa IEB, et al., 2020.

Todos os itens eram utilizados somente uma vez, sendo descartados logo após a finalização das aulas práticas, as atividades foram iniciadas para o primeiro grupo no dia 3 de agosto, conforme apresentado no Quadro 1. As aulas foram realizadas em dois momentos, sendo primeiro voltado para explicação da técnica e cuidados necessários sobre o manuseio dos itens que fariam parte dos procedimentos, e no segundo momento para realização das práticas de enfermagem realizada pelos discentes, sendo colocado em pratica as orientações explicadas pela professora.

O laboratório foi totalmente reformulado para poder receber os alunos de forma segura, o distanciamento foi algo colocado em pratica tanto na explicação, mas como também na realização das práticas. Cada sala continha somente 12 cadeiras, os alunos não poderiam mudá-las de lugar, a professora e os ajudantes ficavam do outro lado do laboratório, que era dividido por vidro transparente de proteção, a comunicação era feita através de microfones conectados entre as salas.

Logo após a explicação da técnica, os alunos eram chamados um de cada vez para realizar o procedimento, a paramentação dos alunos era feita antes de terem contato com os itens e peças disponíveis no laboratório de Suporte Básico de Vida. Iniciando-se com a lavagens das mãos utilizando as técnicas do procedimento, em sequência era solicitado que o aluno calce as luvas de procedimento, coloque a touca, viseira e por último o capote. Após isso, o aluno estava preparado para dar sequência as atividades práticas. 
As práticas de enfermagem eram realizadas em manequins, uma vez que a instituição, por medidas de segurança não autorizava a realização das técnicas em pessoas. Não existia um tempo mínimo para que os alunos pudessem realizar as técnicas, os mesmos poderiam refazer quantas vezes eles considerassem necessárias para sua fixação, em seguida, após a finalização das atividades os alunos descartavam todos os EPIs utilizados e realizavam a lavagem das mãos. Esse mesmo processo foi seguido durante todo o cronograma das aulas no mês de agosto, conforme apresentado no Quadro 1.

\section{DISCUSSÃO}

Um relato de experiência descreve precisamente uma experiência que contribuiu de forma grandiosa para as pessoas envolvidas e principalmente para a sua área de atuação, disponibilizando uma gama de novos conhecimentos adquiridos no decorrer da atividade. Contribuindo assim para a formação acadêmica de muitos outros profissionais (BASTO CC, 2010).

A pandemia causada pela COVID-19 é sem dúvida o maior desafio enfrentado até o momento durante 0 século XXI. A sua alta transmissibilidade, associada a falta de medicamentos e vacinas exclusivas para doença, e um grande risco para população que em muitos casos pode levar as pessoas acometidas à morte, trazendo grandes problemas para saúde pública no mundo (DIAS JAA, et al., 2020).

Por outro lado, percebe-se que o isolamento social possui um horizonte extremante grande, que pode ser visto com diferentes olhares, podendo apresentar aspectos sociais, emocionais, psicológico, cultural e até mesmo espiritual, trazendo uma visão diferente sobre a mesma situação (BITTENCOURT RN, 2020). Para lidar com os impactos causados pelo novo corona vírus, foi necessário realizar algumas mudanças, começando pelo método de ensino e a forma que aquela atividade seria realizada, sempre atento para evitar a disseminação do vírus, através das medidas de prevenção. A pandemia de COVID -19, introduziu o novo normal na vida de milhões de pessoas no mundo, sendo necessário uma readaptação mediante a essa nova situação.

As conexões entre professores, alunos e recursos institucionais tornaram-se menos dependentes da proximidade física, uma vez que o distanciamento social foi colocado em prática, meios como internet, telefones celulares e e-mail contribuíram grandiosamente na educação a distância. Facilitando o acesso as aulas remotas de milhões de pessoas durante esse período de pandemia (TRAXLER J, 2018). Dentro das universidades não foi diferente, foram várias medidas e mudanças necessárias para que os alunos não perdessem o primeiro semestre de 2020. Diante disso, e incrível observar e acompanha os alunos nesse momento tão complicado que o mundo se encontra, mesmo todos passando por uma pandemia, ver o quando eles estavam empenhados em não se atrasarem em relação aos conteúdos daquele semestre e bastante gratificante.

Entretanto, alguns pontos negativos foram percebidos no decorrer das aulas práticas, podemos abordar como principal o medo de se contaminarem com o vírus, uma vez que muitos dos alunos tinham pessoas do grupo de risco em casa, tornando o medo ainda maior. Porém, tanto a instituição como também os alunos observaram o quando era importante o uso de equipamentos de proteção individual para a saúde de todos, evitando assim possíveis riscos de contaminação para eles e seus familiares.

As atividades práticas foram todas realizadas seguindo as recomendações da OMS, visando a saúde e bem-estar de todos as pessoas envolvidas. A instituição dispôs sempre da distribuição de álcool em gel para os alunos, atentando-se todos estavam seguindo as recomendações e utilizando todos os equipamentos de proteção individual. Acredita-se o quando seja ainda importante realizar medidas preventivas para evitar a disseminação do novo coronavírus, algumas atividades não podem ser paralisadas, então cabe as instituições se preparem com todas as medidas e equipamentos necessários para enfrentar a situação da melhor forma possível, sem pôr em risco a vida das pessoas envolvidas.

De acordo com as informações apresentadas, podemos concluir que as práticas realizadas durante a pandemia de COVID-19, foram de extrema importância para o desenvolvimento profissional de acadêmicos como futuro profissionais de enfermagem. Uma vez que, essa experiência possibilitou uma oportunidade de 
absorver uma gama de novos conhecimentos, não somente sobre como lidar com a situação em que estamos vivendo, mas como também em relação a doença, abordando os principais cuidados e medidas preventivas mediante essa situação.

\section{REFERÊNCIAS}

1. CARVER PE, PHILLIPS J. Novel Coronavirus (COVID-19): what you need to know. Workplace Health Saf. 2020; 68(5): 250.

2. MINISTÉRIO DA SAÚDE BR. O que é coronavírus? [Internet]. Brasília: Ministério da Saúde, 2020.

3. GIL A. Como elaborar projetos de pesquisa. Atlas, 2010.

4. HEYMANN DL, et al. COVID-19: what is next for public health?. The Lancet, 2020; 395(10224): 542-545.

5. SEWART D, et al. Distance education: International perspectives. [s.l.] Routledge, 2020.

6. GOULART MB et al. A integração das TDIC na formação inicial de professores de matemática no Brasil: uma análise a partir dos projetos pedagógicos. Olhar de Professor, 2018; 21(2).

7. SILVA AAM. Sobre a possibilidade de interrupção da epidemia pelo coronavírus (COVID-19) com base nas melhores evidências científicas disponíveis. Revista Brasileira de Epidemiologia, 2020; 23.

8. ASSOCIAÇÃO BRASILEIRA DE ENFERMAGEM. Nota da Aben Nacional em relação à ação estratégica "O Brasil conta comigo" [Internet]. Brasília: ABEn, 2020.

9. ALVES L. Educação à distância: conceitos e história no Brasil e no Mundo. Revista Brasileira de Aprendizagem Aberta e à Distância, 2011; 10.

10. GOULART BEH et al. A integração das TDIC na formação inicial de professores de matemática no Brasil: uma análise a partir dos projetos pedagógicos. Olhar de Professor, 2018; 21(2).

11. DIAS JAA, et al. Reflexões sobre distanciamento, isolamento social e quarentena como medidas preventivas da covid-19. Revista de Enfermagem do Centro Oeste Mineiro, 2020.

12. BASTOS CC. Metodologias ativas. 2006. Disponível em: Acesso em: 14 fev. 2010

13. TRAXLER J. Distance learning—Predictions and possibilities. Education Sciences, 2018; 8(1): 35.

14. BITTENCOURT RN. Pandemia, isolamento social e colapso global. Rev Espac Acadêm. 2020; 19(221):168-78. 